\title{
Stem cells in Niemann-Pick disease
}

\author{
Sun-Jung Kim ${ }^{\mathrm{a}, \mathrm{b}, \mathrm{c}}$, Joon-Suk Park ${ }^{\mathrm{a}, \mathrm{b}}$ and Kyung-Sun Kang ${ }^{\mathrm{a}, \mathrm{b}, \mathrm{c}, *}$ \\ ${ }^{a}$ Laboratory of Stem Cell and Tumor Biology, College of Veterinary Medicine, Seoul National University, Seoul, \\ Korea \\ ${ }^{\mathrm{b}}$ Adult Stem Cell Research Center, College of Veterinary Medicine, Seoul National University, Seoul, Korea \\ ${ }^{\mathrm{c}}$ BK2 1 program for Veterinary Science, College of Veterinary Medicine, Seoul National University, Seoul, Korea
}

\begin{abstract}
Neural stem cells are multi-potent and able to self renew to maintain its character throughout the life. Loss of self renewal ability of stem cells prevents recovery or replacement of cells damaged by disease with new cells. The Niemann-Pick type $\mathrm{C} 1$ (NPC1) disease is one of the neurodegenerative diseases, caused by a mutation of NPC1 gene which affects the function of NPC1 protein. We reported that NPC 1 gene deficiency could lead to lack of the self renewal ability of neural stem cells in Niemann pick type $\mathrm{C}$ disease. We also investigated many genes which are involved in stem cells proliferation and differentiation by gene profile in NPC mice.

Diagnosis of NPC disease is difficult because it is accompanied by complicated symptoms and the fact that there is no effective treatment for NPC patients. Studies of these stem cells and their relationship to Niemann pick type C disease will provide new biomarkers for early diagnosis as well as a potential cure by use of targeted therapeutics for Niemann pick type C disease.
\end{abstract}

Keywords: Neural stem cells, niemann pick type C disease, biomarker

\section{Introduction}

Niemann pick type $\mathrm{C}$ disease is characterized by progressive neurodegeneration and arises from mutations in the NPC1 gene on chromosome $18[7,8]$ and is fatal autosomal recessive neurovascular disorder [4] characterized by the progressive neurodegenration of central nerve system, which leads to premature death [3]. Cells lacking functional NPC1 accumulate cholesterol in the lysosomal and late endosomal compartment [41]. At the cellular level, the most prominent feature of the NP-C lesion is lysosomal sequestration of low density lipoprotein (LDL) derived cholesterol, resulting in downstream effects on cholesterol homeostasis [48]. These lipid disturbances are essentially localized to gray matter. Like other lysosomal storage disorder disease, NPC disease shows axonal abnormalities (spheroids, meganeurites, and axonal dystrophy) and demyelination of the corpus callosum [32,

*Corresponding author: Kyung-Sun Kang, D.V.M., PhD, Tel.: +82 2880 1246; Fax: +82 2876 7610; E-mail: kangpub@snu.ac.kr.
44]. NPC 1 gene was identified in 1997 and NPC1 is an integral membrane protein consisting of 1278 amino acids, and NPC 1 residues and cycles mainly late endosome/lysosome and gogli apparatus [7].

The frequency of the Niemann pick diseases type $\mathrm{C}$ is estimated at about 1 in $10^{5}$ live births. The disease occurs more frequently in people of French-Acadian descent in Nova Scotia [47]. Symptoms usually may appear as early as a few months old or as late as adulthood. In childhood on set form, delayed motor development and hypotonia begin between 1 and 2 years of age and most of them die before the age of 5 years [39]. NPC young patients showed enlarged liver and spleen. Enlargement of the spleen and/or liver is associated with an abnormal accumulation of fatty material including cholesterol within the cell. In addition, Niemann Pick type $\mathrm{C}$ disease gradually develop neurological problems include dystonia, dysarthria, learning difficulties, and vertical supranuclear gaze palsy. It is known that the carrier frequency of NPC is about $25 \%$. When both parents are carriers of NPC, the probability of having an affected child increases with each pregnancy. 

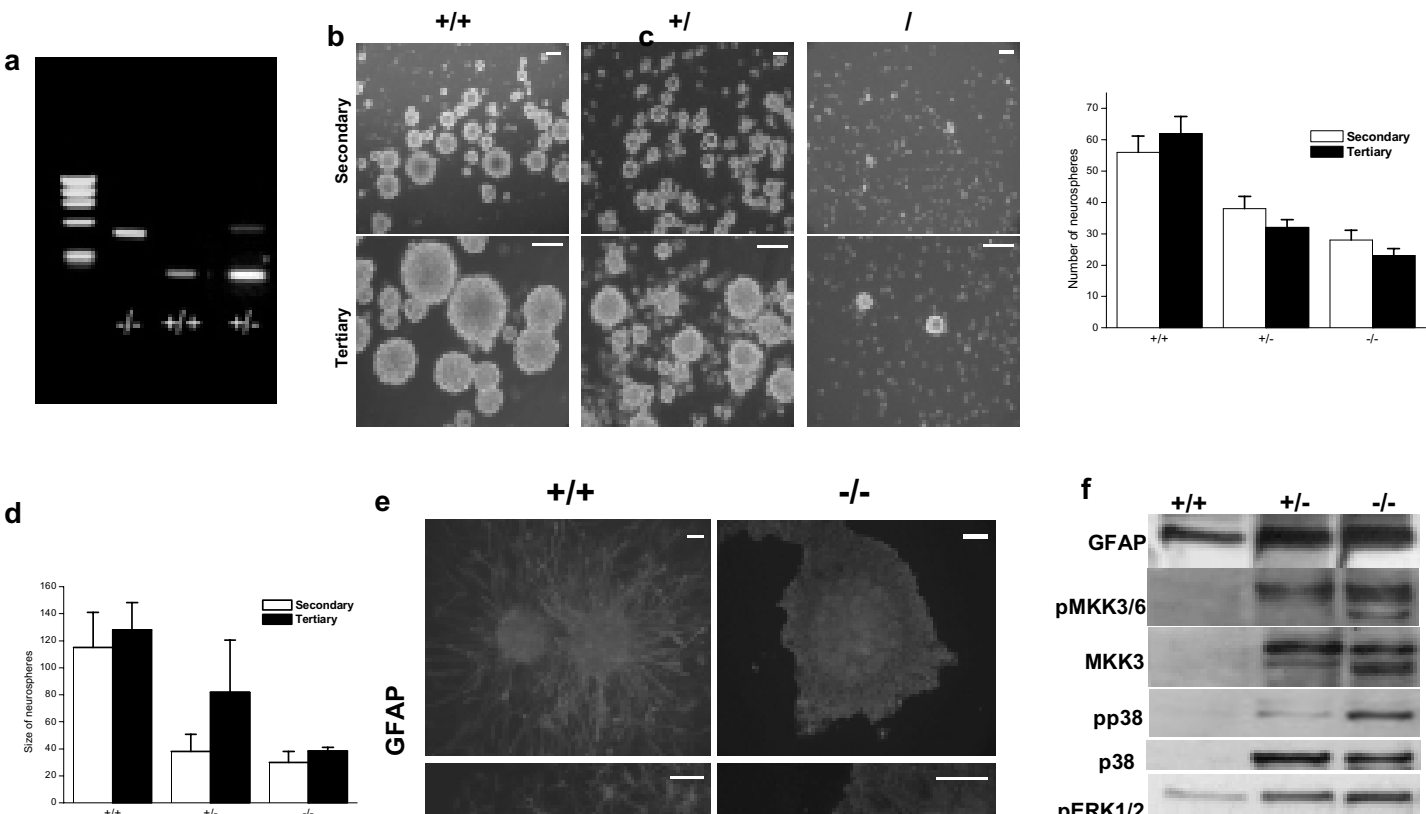

e
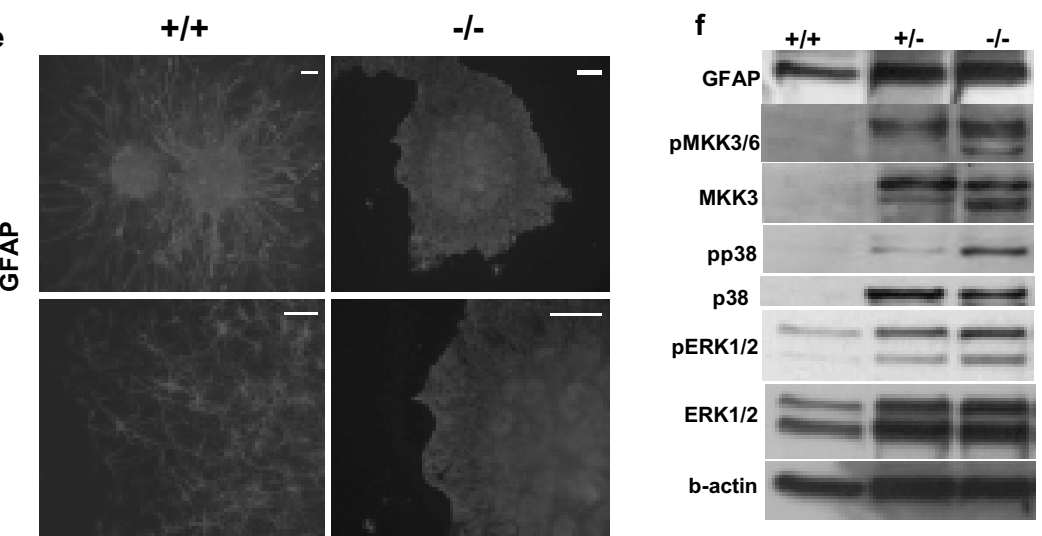

Fig. 1. Neural stem cells (NSC) require NPC to self-renew normally. Images show typical neurospheres that formed after 7d from E16 fetal mice brain. (a) Genotyping analysis with tail-DNA by RT-PCR. (b) Generation of NPC $-/-$ E16 cerebral cells, the cells that formed multipotent neurospheres was significantly reduced relative to wild-type cells. (c-d) Self-renewal capacity is expressed as the number and diameter of secondary and tertiary neurospheres generated per primary neurospheres on subcloning. (e) Generated neurospheres were differentiated by $1 \%$ fetal bovine serum, after $7 \mathrm{~d}$, the spheres were differentiated into astrocytes (GFAP) between wild-type $+/+$ and knock-out $-/-$. (f) Expression of GFAP, pMKK3/6, MKK3, pp38, p38, pERK1/2, and ERK1/2 in wild-type, NPC ${ }^{+/-}$, and NPC ${ }^{-/-}$mice. Values are the mean \pm s.d. for 3-5 independent experiments. Scale bars, $100 \mu$. (Reprinted, from Yang et al., 2006).

\subsection{Stem cells in NPC disease}

Neural stem cells (NSCs) are multipotential progenitor cells that are undifferentiated and capable of proliferation, self-renewal, and the production of many differentiated functional progenies [17]. Moreover, engraftment of these cells leads to recovery in neuropathological conditions $[9,43]$. NSCs possessing the propensity for self-renewal in vitro grow in clonal aggregates called "neurospheres" and may provide an unlimited source of cells for grafting into patients with Parkinson's disease [42], Hungtington's disease [28], and multiple sclerosis [24]. For these reasons, basic studies aiming to well characterize the biology of NSCs are of great interest. These cells are exposed to a high concentration of mitogens such as epidermal growth factor (EGF) or basic fibroblast growth factor (bFGF) and can be induced to differentiate by withdrawing the mitogens or by adding differentiating substances [49]. Behavior of NSCs in culture is affected by multiple variables including: species, growth medium, passage method (dissociated spheres, chopped spheres, and bioreactor) $[2,16]$. Moreover, the ability of immature progenitors and stem cells contained in the in vitro expanded spheres to differentiate in vivo into mature neurons and glia is still unclear. Recently, Milosevic et al. reported spontaneous apoptosis in murine freefloating neurosphere by activation of caspase-3 and Bcl-2 family [30].

Neural stem cells (NSCs) are capable of giving rise to neurons, glia- and astrocytes. Although self renewal and differentiation in neural stem cells are regulated by many genes such as Notch, Numb etc., little is known of the role of defective genes on the self renewal and differentiation of neural stem cells in the developing brain. The Niemann-Pick type C1 (NPC1) disease is one of the neurodegenerative diseases, caused by a mutation of NPC1 gene, which affects the function of NPC1 protein. The ability of NSCs self renewal and differentiation was investigated using a model of Niemann-Pick 
a

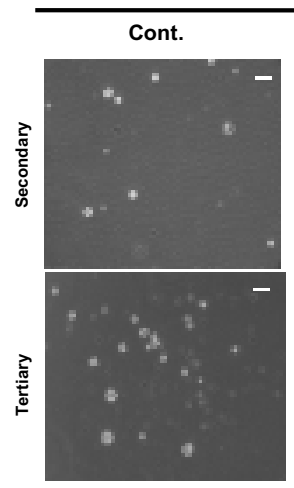

NPC1-/-

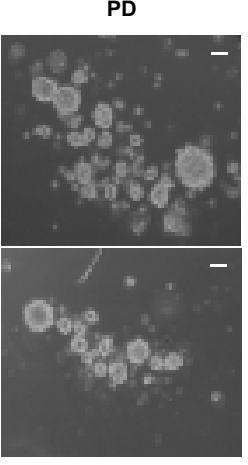

SB

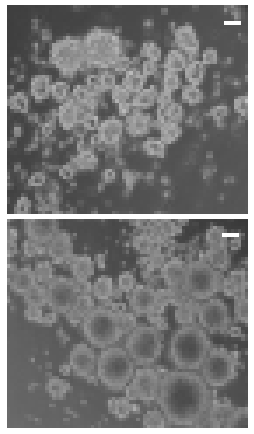

b

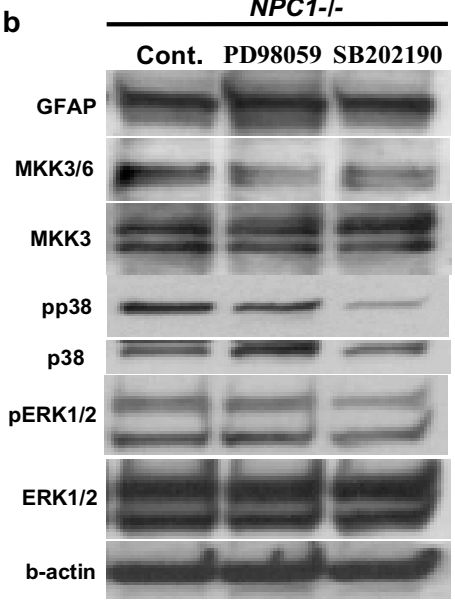

d
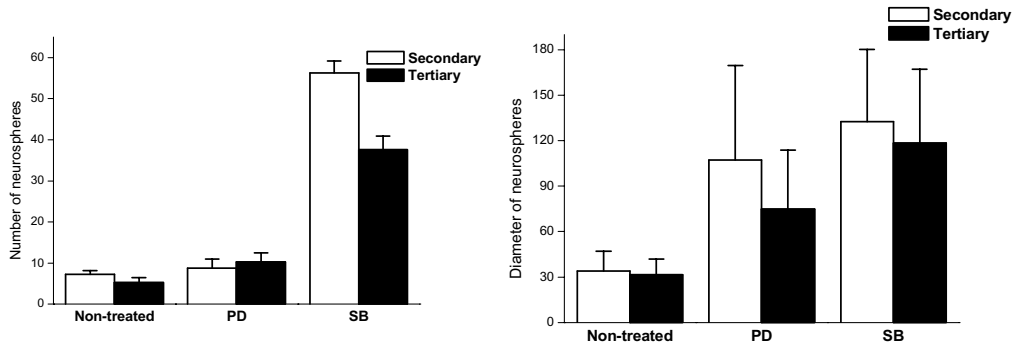

Fig. 2. NPC deficiency increases expression of astrocyte and induces morphological differentiation in astrocyte. (a) Cerebral cells from E16 NPC $-/-$ mice were dissociated and cultured to generate neurospheres in the presence of MEK inhibitor $2 \mu$ M PD98059 and p38 MAP kinase inhibitor $2 \mu \mathrm{M}$ SB202190. (b-c) Self-renewal capacity is expressed as the number and diameter of secondary and tertiary neurospheres generated per primary neurospheres on subcloning. (d) Expression of GFAP, pMKK3/6, pp38 and pERK1/2 were reduced in the neurosphere-derived cells treated with PD98059 and SB202190. Western blotting was done on neurosphere-derived cells (25 $\mu \mathrm{g}$ of protein) from each mouse. Scale bars, $100 \mu$. (Reprinted, from Yang et al., 2006).

type C1 (NPC1) disease. The NPC1 disorder significantly affected the self renewal ability and differentiation of neural stem cells. Neural stem cells from $\mathrm{NPCl}^{-/-}$mice showed impaired self renewal ability when compared to the $\mathrm{NPCl}^{+/+}$mice [50]. These alterations were accompanied by the enhanced activity of p38 MAP kinases. Further, the specific p38 MAP kinase inhibitor, SB202190 improved the self renewal ability of NSCs from $\mathrm{NPC1}^{-/-}$mice (Fig. 2). This indicates that the NPC1 deficiency can lead to lack of self renewal and altered differentiation of neural stem cells mediated by the activation of p38 MAP kinase impairing the generation of neurospheres from $\mathrm{NPCl}^{-/-}$ (Fig. 3). Thus the NPC1 gene may play a crucial role in NSC self-renewal associated with p38 MAP kinase.

\subsection{Biomarkers in NPC}

A biomarker is generally indicator of presence or extent of a biological state that is itself directly linked to the clinical symptoms and indicates particular diseases [22]. Niemann pick type $\mathrm{C}$ showed the heterogeneity due to the onset time or individual differences [34,47]. Many studies have identified biomarkers for Niemann pick disease $[11,14,47]$. In particular, NPC is difficult to diagnose when patients showed typical psychiatric disorder, frontal dementia, ataxia or dystonia and various neurological signs seen in NPC which are similar to other neurological diseases such as Alzheimer's disease, Parkinson's disease and schizophrenia and so on [39]. Therefore, more biological markers are needed to confirm and treat NPC diseases. Previously, we found out NPC1 gene leads to the lack of the self renewal ability of stem cells associated with p38 MAPK kinase. It could be considered as a new biomarker for NPC. Recently, stem cellrelated genes have been proven to be good biomarkers in several diseases such as cancer and cardiovascular diseases. To investigate whether the differences 


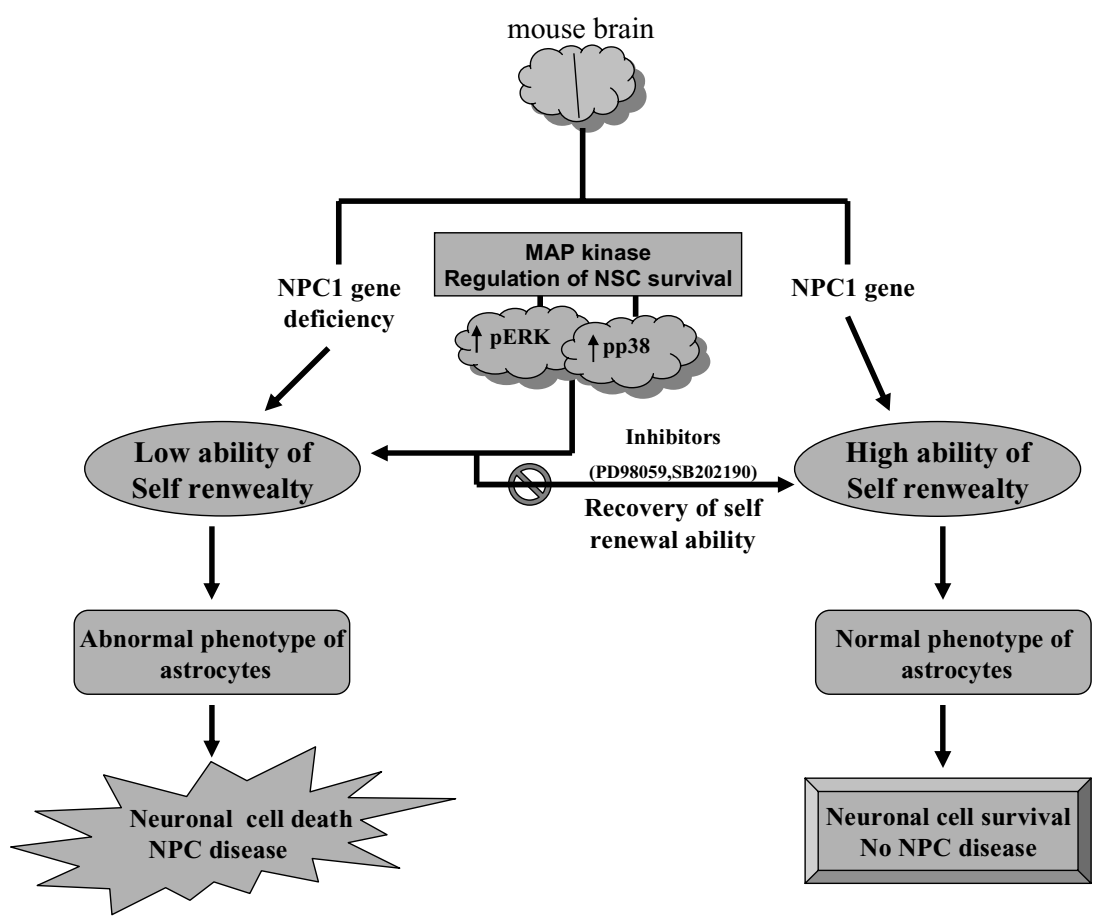

Fig. 3. Schematic diagram for expression and function of NPC1 gene is mediated by MAP kinase in regulation of neuronal stem cell self renewal and differentiation. Neuronal stem cells were isolated from brain of wild type and NPC $-/-$ mice; it was affected to self renewal ability and differentiation of neuronal stem cell not through the MAP kinase (pERK, pp38) depending on NPC1 gene presence. MAP kinase inhibitors, PD 98059(MEK inhibitor), SB202190 (p38 inhibitor) could induce improvement of self renewal ability in neuronal stem cell from NPC1 -/-.

of gene expression profiles of brain ( 8 weeks, hippocampus) derived form NPC1 $-/-$ and $+/+$ mice, we utilized a whole genome bead-based technology (Expression BeadChip for the analysis of $>46,000$ mouse targets based on the Ref Seq, RIKEN FANTOM 2 database, and other data sources) developed by Illumina Inc. that combines the sensitivity and low cost of a focused array with the coverage of a large-scale array [20]. Many more genes (approximately 90\%) were found to be significantly up-regulated than downregulated in NPC1 - / - mice compared with NPC1 $+/+$ mice. The major GO (Gene Ontology) Biological Process terms' relative abundance are illustrated in Fig. 4. NPC1 gene deficiency regulates the expression of genes related to a variety of biological processes. GO biological processes enriched in differentially expressed genes were identified using binominal test [12] as implemented in the Panther Classification System (http://www.pantherdb.org) [46]. The test showed that the genes involved in biological processes such as immunity and defense, developmental processes, cell proliferation and differentiation, T-cell mediated immunity, complement-mediated immunity and MHCI-mediated immunity were significantly enriched $(P<0.05)$ in genes up-regulated in NPC1 $-/-$ mice.
NPC1 gene deficiency also altered the expression of genes related to a broad range of pathways, with angiogenesis, inflammation mediated by chemokine and cytokine signaling pathway, blood coagulation and wnt signaling pathway (Fig. 5). The inset pie chart illustrates the subdivision of the each pathway category.

The most genes were associated with immunity and defense. NPC1 - / - mice derived brain showed higher gene expression values for lectin, galactose binding, soluble 3 (Lgals3, Gal3, GBP, L-34 and Mac-2) and lectin, galactoside-binding, soluble, 3 binding protein (Lgals3bp, 90 K, CyCAP, MAC-2BP, Ppicap) compared to NPC1 $+/+$ mice. Galectin-3, microglia differentiation and differentiation marker, is involved in a variety of biological functions, including cell proliferation and differentiation, inflammation, tumor cell adhesion, angiogenesis, tumor progression and metastasis [6].

Inflammation is mediated by both molecular components, including cytokines, and cellular components, mainly microglia, many of which have pro- and/or antiinflammatory properties [40]. Increased levels of two glial proteins related to the inflammatory response have been observed in Alzheimer's transgenic mice: glial 


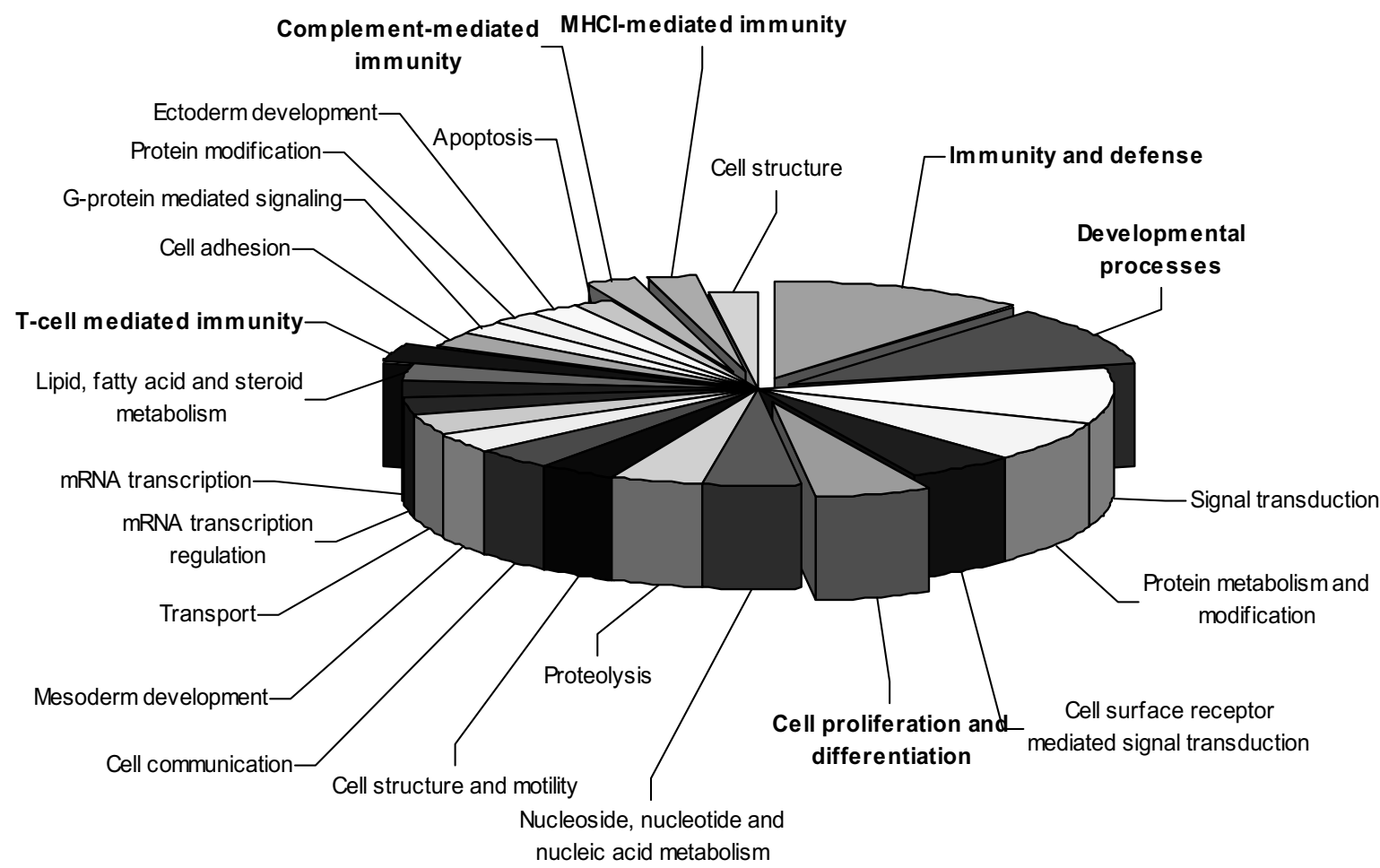

Fig. 4. Major Gene Ontology Biological Process associated with NPC1 gene deficiency. Pie chart indicating GO Biological Process annotations related to NPC1 gene deficiency. Sizes of slices are indicative of probe sets with annotations.

fibrillary acidic protein (GFAP), which overexpression was confirmed by western blotting, and the complement protein C1q. Chronic inflammation has been consistently observed in the brains of $\mathrm{AD}$ patients or transgenic (Tg) mice developing amyloid plaques [10]. Characteristic inflammatory features are the presence of activated microglial cells and reactive astrocytes surrounding plaques, as well as the expression of inflammatory mediators such as cytokines or complement factors [10]. GFAP, an intermediate filament protein specifically expressed in astrocytes, is dramatically up-regulated during reactive astrogliosis [29]. This suggest that astrocyte modifications are more likely to be a consequence of neuronal damage. Our study showed abnormal differentiation of astrocyte phenotype in NPC1-/ - and slightly increased GFAP protein levels but no significant difference between wild type and NPC1 deficient mice [50]. C1q is the initial component of the classical complement pathway, and it can be secreted by both microglia and astrocytes. The absence of $\mathrm{Clq}$ in $\mathrm{Tg}$ mice decreases the level of activated glia without changing the number of amyloid plaques compared to regular Tg animals [15]. The level of both GFAP and C1q was reported to be increased in AD patient brain samples [35,51]. The glycosylation of GFAP was also shown to be increased in AD brains [25]. Moreover, immunochemistry studies showed that GFAP and C1q are concentrated in the surrounding of plaques: GFAP was found in reactive astrocytes, and C1q was observed in microglia and amyloid plaques, both in AD patients brain samples and transgenic mouse brains $[1,26]$. Genes involved in GFAP and several complement components were also up-regulated, suggestion of an alteration in the immunity or/and inflammatory response. Cholesterol accumulation has been a hallmark of the NPC1 disease but the relationship between this accumulation and neurodegeneration remains a mystery. Cholesterol accumulation showed a marked increase in 8 weeks in NPC1 - / - mice, correlating strongly with the development of symptoms [5]. Genes encoding cholesterolrelated proteins (Anxa2, Anxa3 and Cav1) and protein kinase $\mathrm{C}$ were up and down-regulated, respectively, in NPC1 - / - mice. These findings of mRNA level were in agreement with the result of others. Heterozygous and homozygous mice with NPC have an increased expression of caveolin-1 in liver homogenates [18]. Caveolin-1 is responsible for maintaining cholesterol 


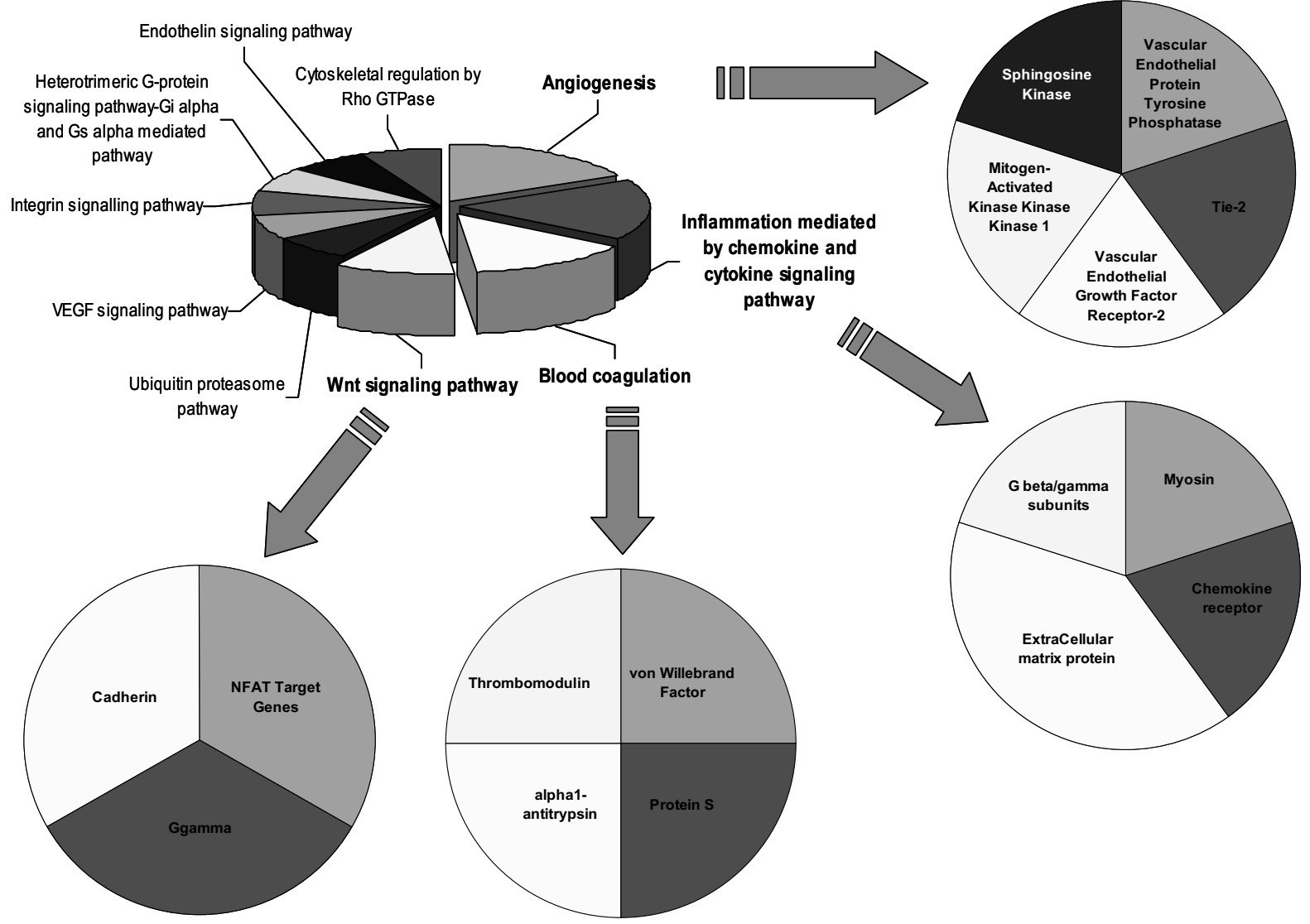

Fig. 5. Major Gene Ontology Pathway associated with NPC1 gene deficiency. Pie charts indicating GO Pathway categories related to NPC1 deficiency. Sizes of slices are indicative of probe sets with that category, and the inset shows the child categories of the GO pathway.

levels by promoting the efflux of excess intracellular unesterified cholesterol. Since the transport of LDLderived cholesterol is impaired in NPC and cells accumulate LDL-derived cholesterol, this excess intracellular cholesterol may result in an increase in caveolin-1 expression. Like caveolin-1, annexin 2 has been found to be associated with caveolae and intracellular vesicles $[33,37,38]$. Annexin 2 is a lipid-binding protein that is widely involved in facilitating intracellular transport, including endocytic trafficking, and is reported to associate with membrane microdomains and normally localizes to early endosomes but has been shown to be mislocalized because of lipid accumulation in late endosomes in NPC disease [13,21,27,31]. Both caveolin1 and annexin 2 contained PKC phosphorylation consensus sequences, although only annexin 2 serves as a direct substrate for PKC $[19,45]$. Caveolin-1 is, instead, serine phosphorylated by casein kinase II $\alpha$ (CK II $\alpha$ ), which is, in turn, activated by PKC [36]. NPC1 deficiency led to lack of self-renewal ability and altered morphology of astrocytes in fetal cerebral cells at day
E16 through the activation of p38 MAPK [50]. Even if gene expression in hippocampus was investigated in 8-week-old NPC mice, genes encoding p38 $\gamma$ and MEKK1, one of $38 \gamma$ upstream, were also slightly upregulated in NPC1 $-/-$ mice. Gene expression analysis of NPC mice has revealed many intriguing similarities with classic neurodegenerative disease. Further study of newly identified candidate genes provide insight into the understanding of the basic mechanism through perturbation of gene networks in a single gene knockout model of NPC disease.

\subsection{Targeted therapeutics and new research in NPC}

Currently, there is no effective treatment for NPC disease patients. Several pharmacological treatments have been used for it to reduce cholesterol accumulation or neurological symptoms or low cholesterol diet is recommended. However, low cholesterol diet or cholesterol lowering drugs didn't change the cholesterol metabolism level or progression of diseases. Gene 
therapy is considered a good treatment for NPC disease but there are many difficulties such as gene delivery technique. In fact, there are no efficient systems to deliver genes to the brain [22]. Recently, one of the promising treatments is stem cell-based therapy. Stem cells have been transplanted into Parkinson disease patients and laboratory models with variable outcomes [23]. Therefore, we can use stem cell therapeutics for NPC disease in the future, possibly using adult stem cells including Umbilical cord blood-derived stem cells and so on. Another therapeutic approach is that there is a link between the lack of self renewal of neural stem cell and some of the neuropathological symptoms seen in NPC1 patients. Thus anti-MAP kinase agents could be of therapeutic importance in patients with the NPC 1 disorder. In our previous work, NPC1 deficiency led to lack of self renewal ability and altered morphology of astrocytes through the activation of p38 MAP kinase, suggesting that p38 MAP kinase inhibitors may be effective method for increasing selfrenewal of neural stem cells for clinical application in NPC1 disease (Fig. 3). It is clear that a greater understanding of the exact mechanism of self-renewal and the function of the NPC1 protein should provide better insights into the application of stem cell therapy in neurodegenerative diseases.

\section{Conclusions}

It is clear that a greater understanding of the exact mechanism of self-renewal and the function of the NPC1 protein should provide better insights into the application of stem cell therapy in neurodegenerative diseases. We conclude that NPC1 gene may be a candidate gene for controlling self-renewal of neural stem cells throughout life - in addition to a pharmacological reagent.

\section{Acknowledgment}

This work was supported by a grant from Korean Science \& Engineering Foundation (R01-2005000-10190-0) and Korean Research Foundation Grant (KRF-005-E00076).

\section{References}

[1] A. Afagh, B.J. Cummings et al., Localization and cell association of C1q in Alzheimer's disease brain, Exp Neurol 138(1) (1996), 22-32.
[2] R.J. Armstrong, P. Tyers et al., Transplantation of expanded neural precursor cells from the developing pig ventral mesencephalon in a rat model of Parkinson's disease, Exp Brain Res 151(2) (2003), 204-2017.

[3] R.O. Brady, M.R. Filling-Katz et al., Niemann-Pick disease types C and D, Neurol Clin 7(1) (1989), 75-88.

[4] T. Bryndorf, B. Christensen et al., Prenatal detection of chromosome aneuploidies in uncultured chorionic villus samples by FISH, Am J Hum Genet 59(4) (1996), 918-926.

[5] K. Byun, J. Kim et al., Alteration of the glutamate and GABA transporters in the hippocampus of the Niemann-Pick disease, type $\mathrm{C}$ mouse using proteomic analysis, Proteomics 6 (4) (2006), 1230-1236.

[6] Z. Cao, N. Said et al., Galectins-3 and -7, but not galectin1 , play a role in re-epithelialization of wounds, $J$ Biol Chem 277(44) (2002), 42299-42305.

[7] E.D. Carstea, J.A. Morris et al., Niemann-Pick C1 disease gene: homology to mediators of cholesterol homeostasis, Science 277(5323) (1997), 228-231.

[8] E.D. Carstea, M.H. Polymeropoulos et al., Linkage of Niemann-Pick disease type $\mathrm{C}$ to human chromosome 18, Proc Natl Acad Sci USA 90(5) (1993), 2002-2004.

[9] P.M. Carvey, Z.D. Ling et al., A clonal line of mesencephalic progenitor cells converted to dopamine neurons by hematopoietic cytokines: a source of cells for transplantation in Parkinson's disease, Exp Neurol 171(1) (2001), 98-108.

[10] I. Casserly and E. Topol, Convergence of atherosclerosis and Alzheimer's disease: inflammation, cholesterol, and misfolded proteins, Lancet 363(9415) (2004), 1139-1146.

[11] C.S. Chen, M.C. Patterson et al., Broad screening test for sphingolipid-storage diseases, Lancet 354(9182) (1999), 901905.

[12] R.J. Cho and M.J. Campbell, Transcription, genomes, function, Trends Genet 16(9) (2000), 409-415.

[13] S. Corvera, C. DiBonaventura et al., Cell confluencedependent remodeling of endothelial membranes mediated by cholesterol, J Biol Chem 275(40) (2000), 31414-31421.

[14] R. Dhami, M.A. Passini et al., Identification of novel biomarkers for Niemann-Pick disease using gene expression analysis of acid sphingomyelinase knockout mice, Mol Ther 13(3) (2006), 556-564.

[15] M.I. Fonseca, J. Zhou et al., Absence of C1q leads to less neuropathology in transgenic mouse models of Alzheimer's disease, J Neurosci 24(29) (2004), 6457-6465.

[16] F.H. Gage, Mammalian neural stem cells, Science 287(5457) (2000), 1433-1438.

[17] F.H. Gage, J. Ray et al., Isolation, characterization, and use of stem cells from the CNS, Annu Rev Neurosci 18 (1995), 159-192.

[18] W.S. Garver, R.P. Erickson et al., Altered expression of caveolin-1 and increased cholesterol in detergent insoluble membrane fractions from liver in mice with Niemann-Pick disease type C, Biochim Biophys Acta 1361(3) (1997), 272280.

[19] K.L. Gould, J.R. Woodgett et al., The protein-tyrosine kinase substrate p36 is also a substrate for protein kinase $\mathrm{C}$ in vitro and in vivo, Mol Cell Biol 6(7) (1986), 2738-2744.

[20] K.L. Gunderson, S. Kruglyak et al., Decoding randomly ordered DNA arrays, Genome Res 14(5) (2004), 870-877.

[21] T. Harder, R. Kellner et al., Specific release of membranebound annexin II and cortical cytoskeletal elements by sequestration of membrane cholesterol, Mol Biol Cell 8(3) (1997), 533-545. 
[22] G. Hsich, M. Sena-Esteves et al., Critical issues in gene therapy for neurologic disease, Hum Gene Ther 13(5) (2002), 579604.

[23] O. Isacson, L.M. Bjorklund et al., Toward full restoration of synaptic and terminal function of the dopaminergic system in Parkinson's disease by stem cells, Ann Neurol 53 Suppl 3 (2003), S135-146; discussion S146-148.

[24] F.P. Jori, U. Galderisi et al., EGF-responsive rat neural stem cells: molecular follow-up of neuron and astrocyte differentiation in vitro, J Cell Physiol 195(2) (2003), 220-233.

[25] K. Kanninen, G. Goldsteins et al., Glycosylation changes in Alzheimer's disease as revealed by a proteomic approach, Neurosci Lett 367(2) (2004), 235-240.

[26] Y. Matsuoka, M. Picciano et al., Inflammatory responses to amyloidosis in a transgenic mouse model of Alzheimer's disease, Am J Pathol 158(4) (2001), 1345-1354.

[27] N. Mayran, R.G. Parton et al., Annexin II regulates multivesicular endosome biogenesis in the degradation pathway of animal cells, Embo J 22(13) (2003), 3242-3253.

[28] J.L. McBride, S.P. Behrstock et al., Human neural stem cell transplants improve motor function in a rat model of Huntington's disease, J Comp Neurol 475(2) (2004), 211-219.

[29] A. Messing and M. Brenner, GFAP: functional implications gleaned from studies of genetically engineered mice, Glia 43(1) (2003), 87-90.

[30] J. Milosevic, A. Storch et al., Spontaneous apoptosis in murine free-floating neurospheres, Exp Cell Res 294(1) (2004), 9-17.

[31] S. Oliferenko, K. Paiha et al., Analysis of CD44-containing lipid rafts: Recruitment of annexin II and stabilization by the actin cytoskeleton, J Cell Biol 146(4) (1999), 843-854.

[32] W.Y. Ong, U. Kumar et al., Neurodegeneration in NiemannPick type C disease mice, Exp Brain Res 141(2) (2001), 218231.

[33] E.T. Parkin, A.J. Turner et al., A role for calcium and annexins in the formation of caveolae, Biochem Soc Trans 24(3) (1996), 444S.

[34] M.C. Patterson, A riddle wrapped in a mystery: understanding Niemann-Pick disease, type C, Neurologist 9(6) (2003), 301310.

[35] G.W. Ross, J.P. O'Callaghan et al., Quantification of regional glial fibrillary acidic protein levels in Alzheimer's disease, Acta Neurol Scand 107(5) (2003), 318-323.

[36] M. Sargiacomo, P.E. Scherer et al., In vitro phosphorylation of caveolin-rich membrane domains: identification of an associated serine kinase activity as a casein kinase II-like enzyme, Oncogene 9(9) (1994), 2589-2595.

[37] M. Sargiacomo, M. Sudol et al., Signal transducing molecules and glycosyl-phosphatidylinositol-linked proteins form a caveolin-rich insoluble complex in MDCK cells, J Cell Biol 122(4) (1993), 789-807.

[38] J.E. Schnitzer, J. Liu et al., Endothelial caveolae have the molecular transport machinery for vesicle budding, docking, and fusion including VAMP, NSF, SNAP, annexins, and GTPases, J Biol Chem 270(24) (1995), 14399-14404.

[39] M. Sevin, G. Lesca et al., The adult form of Niemann-Pick disease type C, Brain 130(Pt 1) (2007), 120-133.

[40] T. Shimizu, H. Imai et al., Cyclophilin C-associated protein and cyclophilin $\mathrm{C}$ mRNA are upregulated in penumbral neurons and microglia after focal cerebral ischemia, J Cereb Blood Flow Metab 25(3) (2005), 325-337.

[41] J. Sokol, J. Blanchette-Mackie et al., Type C Niemann-Pick disease. Lysosomal accumulation and defective intracellular mobilization of low density lipoprotein cholesterol, J Biol Chem 263(7) (1988), 3411-3417.

[42] A. Storch and J. Schwarz, Neural stem cells and Parkinson's disease, J Neurol 249 Suppl 3 (2002), III/30-32.

[43] L. Studer, V. Tabar et al., Transplantation of expanded mesencephalic precursors leads to recovery in parkinsonian rats, Nat Neurosci 1(4) (1998), 290-295.

[44] S. Takikita, T. Fukuda et al., Perturbed myelination process of premyelinating oligodendrocyte in Niemann-Pick type C mouse, J Neuropathol Exp Neurol 63(6) (2004), 660-673.

[45] Z.L. Tang, P.E. Scherer et al., The primary sequence of murine caveolin reveals a conserved consensus site for phosphorylation by protein kinase C, Gene 147(2) (1994), 299-300.

[46] P.D. Thomas, A. Kejariwal et al., PANTHER: a browsable database of gene products organized by biological function, using curated protein family and subfamily classification, $\mathrm{Nu}$ cleic Acids Res 31(1) (2003), 334-341.

[47] M.T. Vanier and G. Millat, Niemann-Pick disease type C, Clin Genet 64(4) (2003), 269-281.

[48] M.T. Vanier and K. Suzuki, Recent advances in elucidating Niemann-Pick C disease, Brain Pathol 8(1) (1998), 163-174.

[49] A.L. Vescovi, B.A. Reynolds et al., FGF regulates the proliferative fate of unipotent (neuronal) and bipotent (neuronal/astroglial) EGF-generated CNS progenitor cells, Neuron 11(5) (1993), 951-966.

[50] S.R. Yang, S.J. Kim et al., NPC1 gene deficiency leads to lack of neural stem cell self-renewal and abnormal differentiation through activation of p38 mitogen-activated protein kinase signaling, Stem Cells 24(2) (2006), 292-298.

[51] K. Yasojima, C. Schwab et al., Up-regulated production and activation of the complement system in Alzheimer's disease brain, Am J Pathol 154(3) (1999), 927-936. 


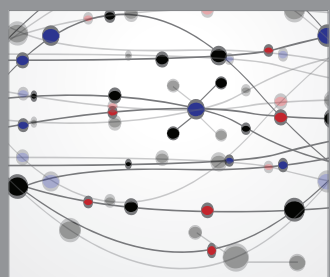

The Scientific World Journal
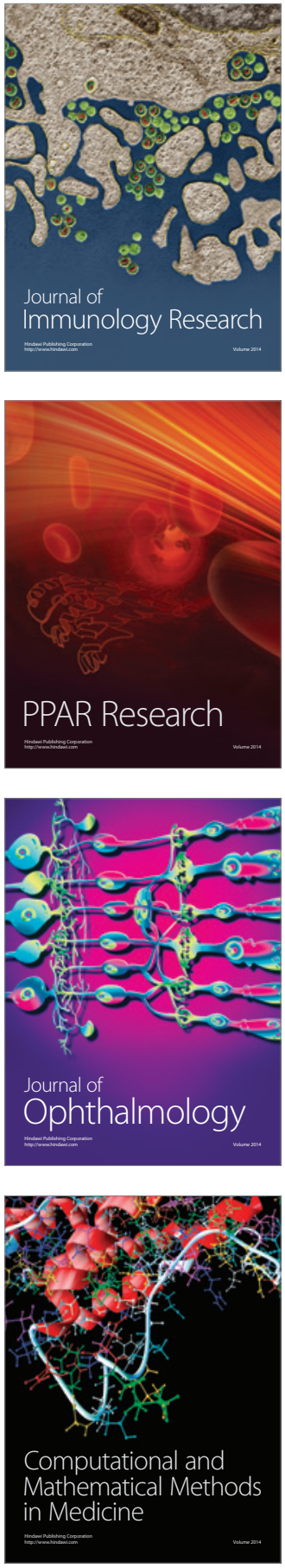

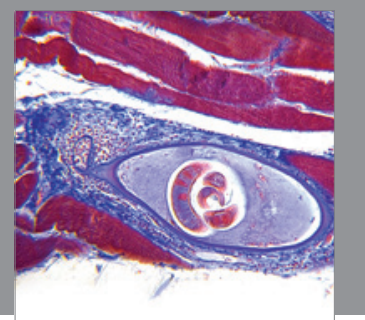

Gastroenterology

Research and Practice
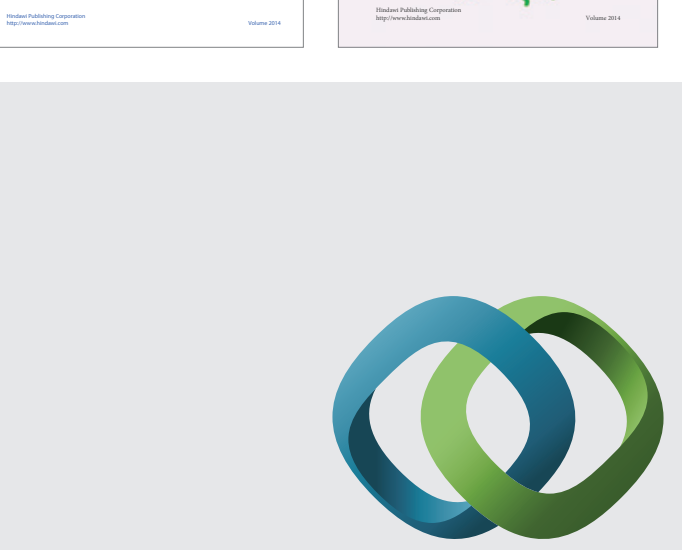

\section{Hindawi}

Submit your manuscripts at

http://www.hindawi.com
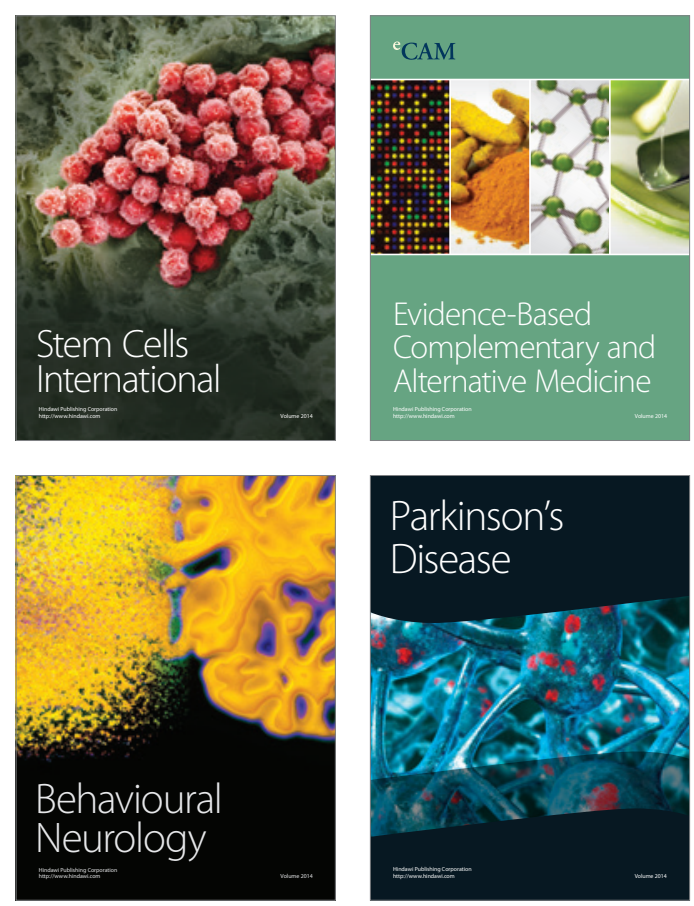

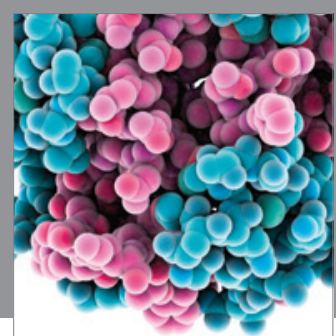

Journal of
Diabetes Research

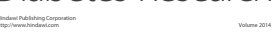

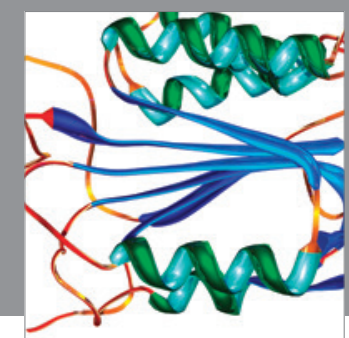

Disease Markers
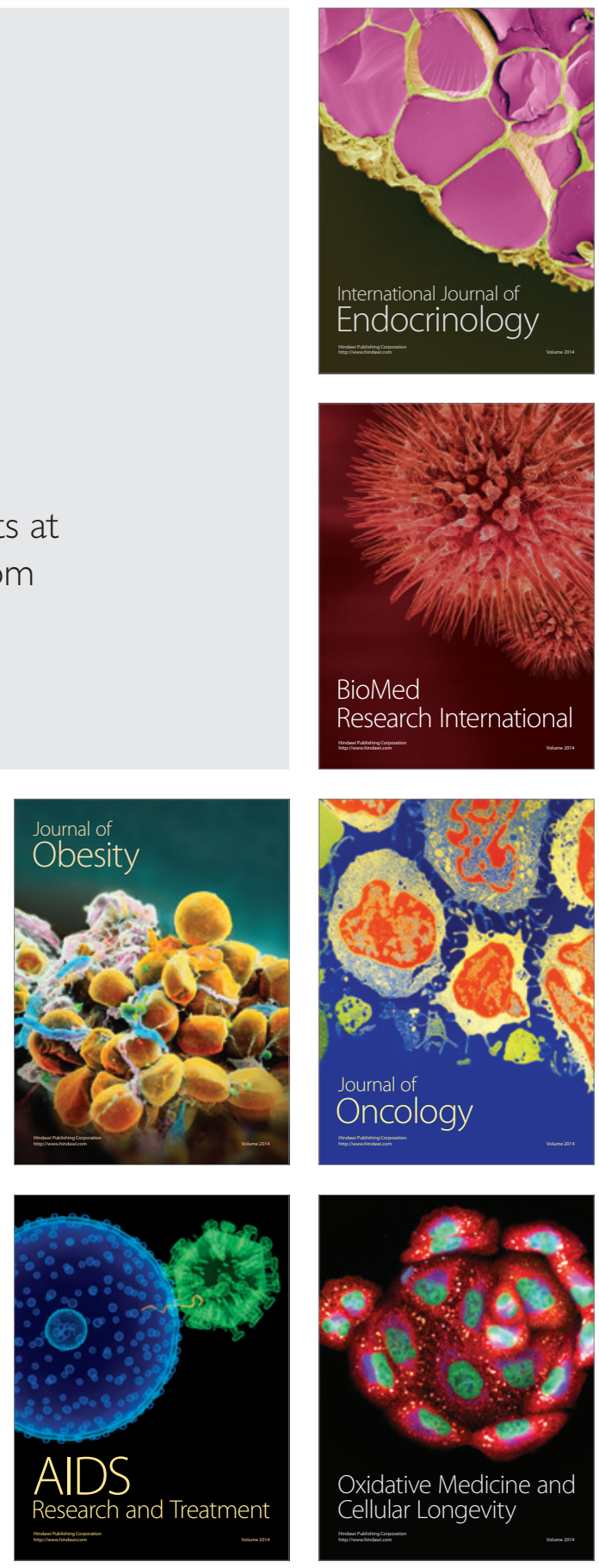